\title{
Fast X-ray reflectivity measurements using an X-ray pixel area detector at the DiffAbs beamline, Synchrotron SOLEIL
}

\author{
Cristian Mocuta, Stefan Stanescu, Manon Gallard, Antoine Barbier, \\ Arkadiusz Dawiec, Bouzid Kedjar, Nicolas Leclercq and Dominique \\ Thiaudiere
}

J. Synchrotron Rad. (2018). 25, 204-213

\section{IU IUCr Journals CRYSTALLOGRAPHY JOURNALS ONLINE}

Copyright (C) International Union of Crystallography

Author(s) of this paper may load this reprint on their own web site or institutional repository provided that this cover page is retained. Republication of this article or its storage in electronic databases other than as specified above is not permitted without prior permission in writing from the IUCr.

For further information see http://journals.iucr.org/services/authorrights.html 


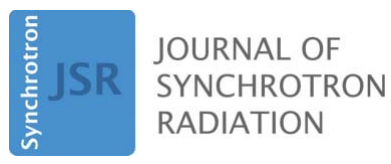

ISSN 1600-5775

Received 28 April 2017

Accepted 27 October 2017

Edited by P. A. Pianetta, SLAC National Accelerator Laboratory, USA

Keywords: X-ray reflectivity; hybrid pixel area detector.

Supporting information: this article has supporting information at journals.iucr.org/s

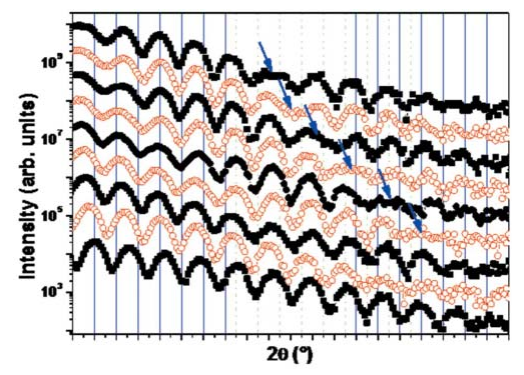

C 2018 International Union of Crystallography

\section{Fast X-ray reflectivity measurements using an $X$-ray pixel area detector at the DiffAbs beamline, Synchrotron SOLEIL}

\author{
Cristian Mocuta, ${ }^{\mathrm{a} *}$ Stefan Stanescu, ${ }^{\mathrm{a}}$ Manon Gallard, ${ }^{\mathrm{a}}$ Antoine Barbier, ${ }^{\mathrm{b}}$ \\ Arkadiusz Dawiec, ${ }^{\mathrm{a}}$ Bouzid Kedjar, ${ }^{\mathrm{c}}$ Nicolas Leclercq ${ }^{\mathrm{a}}$ and Dominique Thiaudiere ${ }^{\mathrm{a}}$
}

${ }^{\text {a}}$ Synchrotron SOLEIL, L'Orme des Merisiers, Saint-Aubin, BP 48, Gif-sur-Yvette 91192, France, bDSM/IRAMIS/SPEC, CEA Saclay, Gif-sur-Yvette 91191, France, and 'Institut Pprime, CNRS - University of Poitiers - ENSMA, SP2MI, Futuroscope 86962, France. *Correspondence e-mail: mocuta@synchrotron-soleil.fr

This paper describes a method for rapid measurements of the specular X-ray reflectivity signal using an area detector and a monochromatic, well collimated X-ray beam (divergence below $0.01^{\circ}$ ), combined with a continuous data acquisition mode during the angular movements of the sample and detector. In addition to the total integrated (and background-corrected) reflectivity signal, this approach yields a three-dimensional mapping of the reciprocal space in the vicinity of its origin. Grazing-incidence small-angle scattering signals are recorded simultaneously. Measurements up to high momentum transfer values (close to $0.1 \mathrm{~nm}^{-1}$, also depending on the X-ray beam energy) can be performed in total time ranges as short as $10 \mathrm{~s}$. The measurement time can be reduced by up to 100 times as compared with the classical method using monochromatic $\mathrm{X}$-ray beams, a point detector and rocking scans (integrated reflectivity signal).

\section{Introduction}

Specular X-ray reflectivity (XRR) is a well established and powerful technique for thin film characterization. It gives easy access to important average information like thickness, electron density and roughness of thin films (on a substrate). These quantities are probed in a direction perpendicular to the surface of the sample. The relatively large penetration depth (range of several micrometres) of hard X-rays (of several $\mathrm{keV}$ energy) gives access to such quantities not only for thin films at the outermost surface but also for buried interfaces. Provided that a correct and appropriate modelling of the experimental data is carried out (Stepanov, 2017; Fujii, 2010, 2013), a resolution in the $0.1 \mathrm{~nm}$ range can be expected. The precision is improved by using data measured up to large momentum transfer $(q)$ values and including the contribution of the diffuse scattering as well (Sinha et al., 1988; Weber \& Lengeler, 1992). Experimentally, the fast decay of the Fresnel reflectivity signal in the reciprocal space (as $q^{-4}$ ) requires high signal-to-noise detection and intense X-ray sources, as available at synchrotron facilities. In such cases, intensity values spanning over up to ten orders of magnitude can be accessed during the measurement of the XRR curve and access to large $q$ values (several $0.1 \mathrm{~nm}^{-1}$ ) is possible. Indeed, measuring the XRR curve as far as possible in the momentum transfer $q$ space is needed for a robust modelling of the data, allowing one to show, for example, the presence of ultra-thin layers (down to single monolayers). Also, in particular cases, the measurement of the off-specular (diffuse) or small-angle scattering signals gives information about the islands' distri- 
bution, lateral roughness, surface layer and/or interface morphology (i.e. in a direction parallel to the sample surface) etc. Most of the time, these signals are of very low intensity (up to several orders of magnitude lower compared with the XRR signal) and require the use of intense and well collimated beams as provided by the synchrotron source.

Recently, much effort has been put into optimizing and speeding up the XRR acquisition schemes. In a classical measurement scheme, the acquisition of an XRR curve takes from several minutes (in the best cases for qualitative measurements) to several hours (as we will detail later in this paper) when quantitative background-corrected and integrated scattered data are considered. These time periods are far too long in most cases to gain insight into the dynamics and evolution phenomena of thin film samples at their surfaces or interfaces. Moreover, it might be difficult to ensure that the sample is maintained in its original state (i.e. clean and unchanged in its local environment) in the X-ray beam over extended time periods, such as those required by the classical XRR measurement scheme. This condition is even more critical for soft condensed matter thin films.

Most of the existing XRR studies (see details further in the text) stress the need for fast XRR measurements (Kobayashi \& Inaba, 2012): a faster acquisition scheme grants access to detailed studies of time evolution of chemical, thermal or mechanical changes at surfaces and interfaces. Each such phenomenon relies on a characteristic time scale which needs to be accessed by an adapted XRR data acquisition speed. We will show in this paper that a time resolution of up to $10 \mathrm{~s}$ can give valuable information in such studies.

The experimental approach proposed hereafter uses: (i) a monochromatic low-divergence (below $0.01^{\circ}$ ) X-ray synchrotron beam; (ii) a fast-reading, high-dynamics (up to six orders of magnitude) and low-background area detector; (iii) a continuous acquisition mode of the data, realized during the synchronous angular movement of the sample and detector (Mocuta et al., 2013; Medjoubi et al., 2013; Leclercq et al., 2015). The obtained data are then reconstructed to extract not only a background-corrected and integrated XRR signal but also a full three-dimensional data set in the reciprocal space. We will show the important gain in acquisition time of betterquality XRR data sets, compared with the point detector stepby-step approach. Data on the same sample using these two approaches will be shown and compared.

Our setup offers flexibility in terms of: (i) X-ray beam energy (avoiding absorption edges of chemical elements making up the sample, i.e. reducing fluorescence background); (ii) angular (and thus $q$-space) resolution (which can be adapted, depending on the film/multilayer thickness); (iii) exposure time per image (i.e. detection of low signals at high $q$ values will require longer exposure times than the beginning of the XRR curve; the consequence might be, of course, detrimental to a fast XRR acquisition of the full curve); and (iv) usage of a classical diffractometer setup.

The paper is organized as follows: after the description of the samples investigated in this study, the use of the area detector combined with the continuous acquisition mode is detailed. Comparison between data obtained with these two approaches will be shown on a model (multilayer) sample. The supporting information obtained when performing the fast XRR measurement will be highlighted. The power of the method will be exploited via two more examples in which some kinetics are addressed. The first one is of a sample which evolves under X-ray illumination. The second one concerns the characterization of a diffuse interface during its formation in a thermal annealing process. Triggering and measuring such phenomena required rapid measurements of the XRR curve over extended $2 \theta$ angular (or momentum transfer $q$ ) ranges. Finally, the advantages and limitations of the method will be highlighted, discussed and compared with previously reported fast acquisition XRR methods. Possible research and analysis fields in which this method could shed new light and allow useful information to be obtained will also be discussed. A brief reminder of the XRR principle and classical (point detector) measurement setups (using monochromatic X-ray beams), together with other possible XRR acquisition schemes, is given in the supporting information.

Despite the numerous advantages that the methods detailed in the supporting information bring to the XRR technique, they also suffer from a number of limitations including rather complex setups which offer less flexibility in terms of maximal accessible momentum transfer values, limited possible sample environments or an uneasy tuning of the (angular or $q$-space) resolution of the acquired data. Moreover, if the energy range spans over one or several absorption edges of the materials used in the sample, the fluorescence signal might be detected and contribute as background only to the XRR measurement.

\section{The samples}

Three samples were examined in this study to illustrate the validity of our fast XRR approach using an area detector (XPAD, X-ray pixel area detector). The examples were also selected to highlight scientific cases for which fast and quantitative (integrated) XRR measurements of the scattered signal are of the utmost importance to understand and characterize the structure of the samples. The data naturally contain complementary grazing-incidence small-angle scattering (GISAXS) data that will also be exploited and discussed.

\section{1. 'Soliton' sample}

In order to validate the approach, similar XRR data were recorded and compared with both a point and area detector. The sample used consists of several metallic layers and its structure is depicted in Fig. 1 (inset): a soft magnetic layer of $\mathrm{CoFeB}$, less than $1 \mathrm{~nm}$ thickness, is grown as a sandwich between two Pt layers. In order to ensure optimal growth conditions for the bottom Pt layer on the Si wafer, a buffer Ta layer is used. While scientific interest in this sample is due to it being part of complex architectures acting as a spintronic unidirectional vertical shift register (Lavrijsen et al., 2013), it 


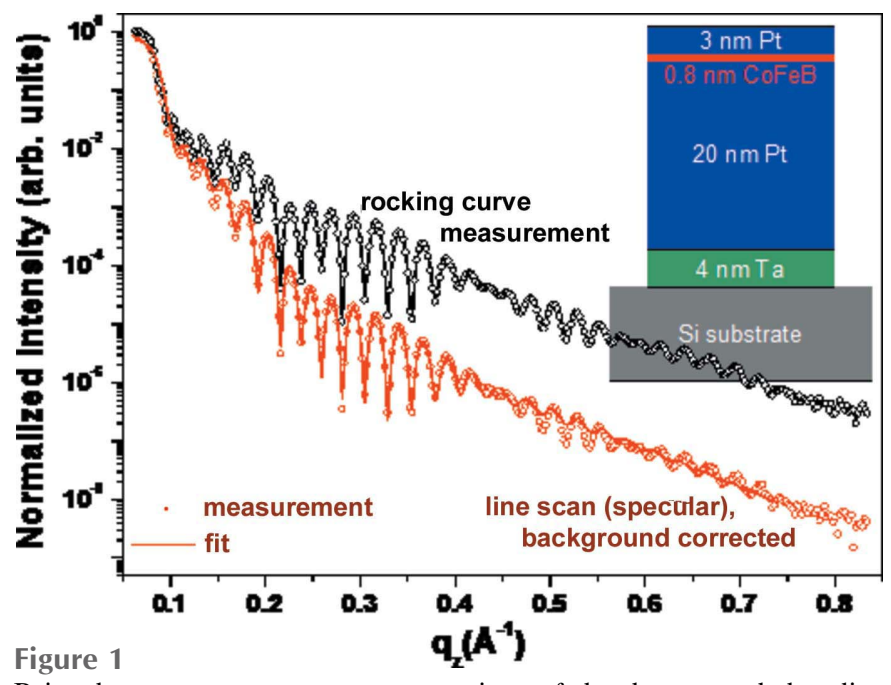

Point detector measurements: comparison of the data recorded as line scans in specular condition with background subtraction and the curve recorded as a result of rocking scans at each point. This last measurement took about $8 \mathrm{~h}$ ( $1 \mathrm{~s}$ exposure per point). In one case, the data are also simulated and fitted to retrieve the thickness and roughness of the layers (see text for details). Note, for the last mentioned data, the interference at $q \sim 0.17 \AA^{-1}$ which is much more visible. (Inset) The structure of the soliton sample (the thickness and materials of the different layers).

is simply used here as a model sample, validating our experimental approach.

\subsection{Thin epitaxial film of $\mathrm{BaTiO}_{3}$ on $\mathrm{SrTiO}_{3}$}

A second sample illustrates the need for reliable and fast XRR measurements. It became evident, during this study, that, for this second sample, its thin film structure evolves while the sample is being illuminated by the X-ray beam. A fast XRR approach which guarantees corrected and integrated intensities is required to follow this effect and to make sure it is not an artifact, as will be shown later in this paper. The sample consisted of an epitaxial thin film ( $\sim 10 \mathrm{~nm}$ thick) of $\mathrm{BaTiO}_{3}$ deposited on a single-crystalline $\mathrm{SrTiO}_{3}(001)$ substrate, using atomic oxygen assisted molecular beam epitaxy in a chamber containing dedicated $\mathrm{Ba}$ and Ti Knudsen cells (for details, see Barbier, Mocuta, Stanescu et al., 2012; Barbier et al., 2015). We demonstrated in a previous study (Barbier, Mocuta, Stanescu et al., 2012) that the $\mathrm{BaTiO}_{3}$ film grown on the $\mathrm{SrTiO}_{3}$ substrate is two-dimensional, epitaxial, and that the interface is sharp. These films are ferroelectric and have great potential in the context of developing nonvolatile random access memories and novel applications in the field of spintronics using multiferroic systems. The sample mentioned here can be seen as a model one. Apart from its stability (oxide sample), $\mathrm{BaTiO}_{3}$ has been known for decades for being the prototype ferroelectric perovskite-type material. Moreover, Fe doping of the layer revealed recently the coexistence of ferroelectricity and magnetic long-range ordering (Barbier et al., 2015; Xu et al., 2009).

\subsection{Thin Ni film on Si}

The third example deals with the in situ investigation of the formation of a diffuse interface as may occur during a thermal annealing of a thin layer (Putero et al., 2010; Ehouarne et al., 2006). A $20 \mathrm{~nm}$-thick Ni layer deposited on single-crystalline $\mathrm{Si}(001)$ substrate was used as a test sample. The layer is capped by a $5 \mathrm{~nm}$-thick TiN layer to prevent oxidation. It is important to follow, during the annealing of the sample, not only the formation of such an interface (e.g. thickness) but also the build up of stress as this can affect properties like continuity of the layer, growth of the diffuse interface etc. Nickel-silicon systems may be an alternative in microelectronics (Lavoie et al., 2003; Kittl et al., 2003; Breil et al., 2015) to form very thin ohmic contacts on Si (Lauwers et al., 2004; Imbert et al., 2010).

\section{Experimental setup and data acquisition approach}

In the following, we propose the use of a monochromatic $\mathrm{X}$-ray beam, a continuous acquisition scan mode (Medjoubi, Bonissent et al., 2013; Medjoubi, Leclercq et al., 2013; Mocuta et al., 2013; Leclercq et al., 2015) and an area detector (XPAD) having a high sensitivity, large dynamic range, low noise and fast response (Delpierre et al., 2007; Medjoubi et al., 2010, 2012; Fertey et al., 2013, and references therein).

The experiments described in this paper were all performed at the French synchrotron source (Synchrotron SOLEIL, DiffAbs beamline, https://www.synchrotron-soleil.fr/en/beam lines/diffabs). In all the cases depicted here, an X-ray energy in the 7.0 to $19.0 \mathrm{keV}$ range was used; the precise value will be detailed for each measurement. The beam size was about 200 $\times 250 \mu \mathrm{m}$ [full width at half-maximum $(\mathrm{FWHM})$, vertical $\times$ horizontal]. The beam divergence in vertical (V) and horizontal $(\mathrm{H})$ directions amounted to $0.01^{\circ}$ and $0.09^{\circ}$, respectively. The XRR experiments were carried out with a horizontal sample surface; thus the scattering plane is vertical.

The detector used for this study was the XPAD S-140 hybrid pixel detector. The family of XPAD3 photon-counting readout chips has been extensively described elsewhere (Basolo et al., 2007; Pangaud et al., 2007, 2008); therefore only its main characteristics are presented in this paper. The circuit comprises 9600 square pixels with a pitch of $130 \mu \mathrm{m}$, organized in a matrix of 80 rows and 120 columns. Each pixel is equipped with a 12 bit counter with an extra bit that can be accessed during exposure; this allows the dynamic range to be extended up to 32 bits. The response of the detector is linear up to $3 \times 10^{5}$ photons s$^{-1}$ pixel $^{-1}$ and photon selection is done with a single threshold discriminator. The minimum threshold that can be set corresponds to an energy of $4 \mathrm{keV}$ (Basolo et al., 2008). The XPAD S-140 detector is made up of 14 XPAD3.2 chips bump-bonded to a single $500 \mu \mathrm{m}$-thick silicon sensor. The XPAD circuits are organized in two rows of seven chips giving about $75 \times 32 \mathrm{~mm}$ of sensitive surface $(240 \times 560$ pixels). The sensor is pixelated with the same pitch as the readout circuits except for the zones that correspond to the gaps between adjacent chips that are 3 pixels wide. Furthermore, the detector offers fast readout (depending slightly on the chosen dynamic range of 16 and 32 bits, in the range of a few $\mathrm{ms}$ ) and low noise (not influenced by the background 
noise). These specifications make this detector a very interesting option for XRR measurement.

To ensure that all pixels in the matrix will respond uniformly to photons of a given energy, the discriminator threshold of each pixel must be calibrated individually. This is done in a two-step process; first a circuit global threshold is adjusted that is later fine-tuned in each pixel in order to compensate dispersion of the microelectronic process. The threshold should be set at half the working energy in order to avoid a charge sharing effect. For some of these measurements (X-ray beam energy in the 7.0 to $8.0 \mathrm{keV}$ range) the minimum threshold was below half of the working energy; thus it was set above the noise peak of each pixel.

A geometrical distortion correction for the pixel position might be performed, similarly to the one for fibre-optic tapers in X-ray CCD detectors (Paciorek et al., 1999a,b); it can possibly be the result, for example, of a non-accurate mechanical mounting of the XPAD module(s) in the detector frame. It was not necessary for this study, but could be performed by using an absorbing mask with small holes placed on a regular square grid. The geometrical effect of the largersize pixels was corrected in the data treatment procedure (Mocuta et al., 2013; Le Bourlot et al., 2012).

After sample alignment (sample placed at the centre of rotation of all the circles of the diffractometer, and coinciding with the incoming beam), changing the incident angle of the sample $\alpha_{\mathrm{i}}$ is still required to measure points along the $q_{z}$ direction. The area detector covers a certain $\alpha_{\mathrm{f}}$ range; however we keep its angular position so as to maintain $\alpha_{\mathrm{f}}=\alpha_{\mathrm{i}}$. Each image acquired with the area detector represents a twodimensional cut (but not by a plane) in the reciprocal space. The whole measurement will thus yield a three-dimensional data set in reciprocal space, in the region close to its origin $(q=0)$ (see Fig. S2 in the supporting information). The measurement implies the continuous scanning of the angular position of the sample and, possibly, of the detector. If the detector angle is scanned as well, the two motors are moving synchronously with adapted speeds and accelerations so as to have $\alpha_{\mathrm{f}}=\alpha_{\mathrm{i}}$. The XPAD images are acquired during the movement, at high acquisition speed (e.g. possible up to a repetition rate of several $100 \mathrm{~Hz}$ in some very specific configurations). Thus one image corresponds to an integration of the scattered signal over a certain angular range. The exposure time per image and the motor speed are chosen in order to obtain both the required angular resolution and counting statistics. The gain with this measurement approach is twofold: on the one hand, dead times are reduced down to a minimum by removing the motors' acceleration/deceleration/ stop times (as in step-by-step scanning), and, on the other hand, it provides a complete three-dimensional mapping of the reciprocal space (i.e. integrated scattered signal and background correction recorded in the same data set).

In the continuous motor movement acquisition scheme, a $2 \theta$ range of $10^{\circ}$ can be covered with $0.01^{\circ}$ angular resolution and a $10 \mathrm{~ms}$ exposure time per point in around $10 \mathrm{~s}$ of total measurement time. If the dynamic range of the detector is not large enough to measure the full XRR curve, calibrated beam attenuators can be used. In this case the proper angular range has to be determined in order to use the right beam attenuation (to ensure a linear response of the detector) - the XRR curve will be cut in several $q$ ranges and reconstructed after the attenuation correction. The use of attenuators will introduce some additional sub-second dead times for each scanned angular range by the motors' acceleration/deceleration and insertion of automatic attenuators in the beam.

The recorded XPAD images are converted into $q$-space data using the proper geometry transformation (see, for example, Mocuta et al., 2008, 2013; Schlepütz et al., 2005). A three-dimensional data set is generated. We will show in the following the different types of information which can be extracted. For comparison, similar XRR data were recorded, on the same sample, using a point detector (Fig. 1). The slits defining the detector aperture were set at $0.25 \times 1.3 \mathrm{~mm}(\mathrm{~V} \times$ $\mathrm{H}$ ) opening, yielding an angular aperture (during the point detector measurement) of $\sim 0.03^{\circ} \times 0.15^{\circ}(\mathrm{V} \times \mathrm{H})$.

\section{Results and discussion}

We mentioned earlier in this paper the use of point detectors for XRR measurements, with different approaches; the two approaches (background correction using an 'offset' line scan and integrated intensities extracted from rocking scans) are compared for the multilayer ('soliton') sample, under the same measurement conditions (same experiment session) in Fig. 1. The data were normalized to the same value for the total external reflection plateau. One can note a slightly better defined wide oscillation around $q \simeq 0.17 \AA^{-1}$ in the case of rocking-curve-extracted data. The XRR curve can also be simulated and fitted; such a result is shown in Fig. 1, yielding thicknesses slightly lower than - but still in agreement with the ones expected for this sample: $2.8 \mathrm{~nm} \mathrm{Pt} / 0.9 \mathrm{~nm} \mathrm{CoFeB/}$ $18.9 \mathrm{~nm} \mathrm{Pt} / 3.1 \mathrm{~nm} \mathrm{Ta} / \mathrm{Si}(001)$. The interfacial roughness is found to be below $0.5 \mathrm{~nm}$ except for the $\mathrm{CoFeB} / \mathrm{Pt}$ interface, which amounts to slightly more than $2 \mathrm{~nm}$.

The sample was also measured using the XPAD detector. The set of data reported hereafter was acquired at an X-ray energy of $7.0 \mathrm{keV}$.

From the data set containing the XPAD images, it is possible to reconstruct the equivalent of a point detector measurement. This was done using Python (https:// www.python.org/) specific code. The conversion of the data sets in $q$-space coordinates as well as the various two-dimensional cuts along high-symmetry $q$ directions were performed using open-source ImageJ (https://imagej.nih.gov/ij/) macros. The three-dimensional visualization of the data set was performed using open-source Paraview software (https:// www.paraview.org/). The source codes used can be found in the supporting information.

In practice, a region of interest (ROI) is defined on the XPAD image at the expected (theoretical) position of the reflected beam (geometrical condition $\alpha_{\mathrm{i}}=\alpha_{\mathrm{f}}, c f$. Fig. S1). The size of this ROI was chosen to be similar to the angular opening of the point detector slits $(0.25 \times 1.3 \mathrm{~mm} \mathrm{~V} \times \mathrm{H}$, i.e. $\sim 2 \times 10$ pixels) during the XRR measurement. On the 
same image a background signal can be extracted by simply shifting the same ROI in the reciprocal space (i.e. on the threedimensional data set). The agreement with the corresponding point detector XRR data is very good, as expected. One may note anyhow that in this case the background is not equivalent to that recorded using rocking scans with the point detector: the background with the XPAD is taken at a different $q_{y}$ value, while with the point detector (rocking scan) a different $q_{x}$ is accessed. It can also be noted here that a background similar to the point detector rocking-curve measurement can be extracted as well from the three-dimensional data. In the rocking-scans point detector approach, the resulting total scattered intensity is integrated along one direction only (namely $q_{x}$ ) in the reciprocal space. We should point out here that the use of a pair of slits in front of the point detector will better suppress on the XRR signal the diffuse scattering by defining a real angular acceptance: the detector 'sees' only the active area of the sample. This is clearly not the case when using an ROI on the area detector: each pixel can see diffuse signal originating from areas of the sample illuminated by the $\mathrm{X}$-ray beam and which are not in the centre of the sample. The XRR data extracted from the three-dimensional data set acquired with the XPAD comprise a two-dimensional integration in the $\left(q_{x}, q_{y}\right)$ plane (Fig. S2). The three-dimensional reconstructed reciprocal-space volume also allows extraction of the signal along the line $q_{x}=$ constant, which can be used as background to be subtracted from the XRR data (Fig. S2). This might explain the difference highlighted in Fig. 2 (left panel) - the broad oscillation around $q \simeq 0.17-0.2 \AA^{-1}$ is even more visible. The XRR data are obtained from the threedimensional data set by integration (summation) over the pixels containing non-background data. This ROI can be adapted in the data set at any time, which is not possible for the point detector measurements - in which case the slit settings might not always be adapted, but keep in mind that a better background suppression can be done only using the pair of slits. Data binning can also be performed to adapt the results to the required resolution and increase the counting statistics as well. Also, the three-dimensional data set allows one to rule out (from the integrated and corrected XRR data) the presence of some parasitic signals like diffuse scattering, Yoneda peaks etc. In the classical XRR acquisition scheme using a point detector, such parasitic signals can be measured and misinterpreted as true (specular) contributions. It can though be minimized by a proper setting of the detector slits (typically closing them) at the cost of limited counting rates, but having an optimized opening (in terms of counting statistics and background removal) is not straightforward.

Once the XPAD data are reconstructed (in three dimensions), cuts along high-symmetry directions (namely the planes $q_{x}=0$ and $q_{y}=0$, Fig. S2) can be examined. The first one corresponds to the so-called GISAXS region (see, for example, Renaud et al., 2009; Barbier, Mocuta, Belkhou et al., 2012). We should point out here that, in most cases, a GISAXS measurement consists of a single image recorded with an area detector, placed to record the X-ray scattered signal close to the direct (transmitted) X-ray beam, i.e. the vicinity of the $q=$ 0 region of the reciprocal space. The assumption of a relatively flat Ewald sphere is made (large X-ray beam energy, of several $\mathrm{keV}$ ), so that the image obtained directly corresponds to the $q_{x}=0$ slice in the reciprocal space. While this assumption holds in most cases when broad scattered signals are expected (for example, nanostructured as-grown surfaces), it is not valid in the case of a well structured sample [e.g. artificial gratings; see, for example, Yan \& Gibaud (2007), Stanescu et al. (2013)]. In such cases, even the very small curvature of the Ewald sphere
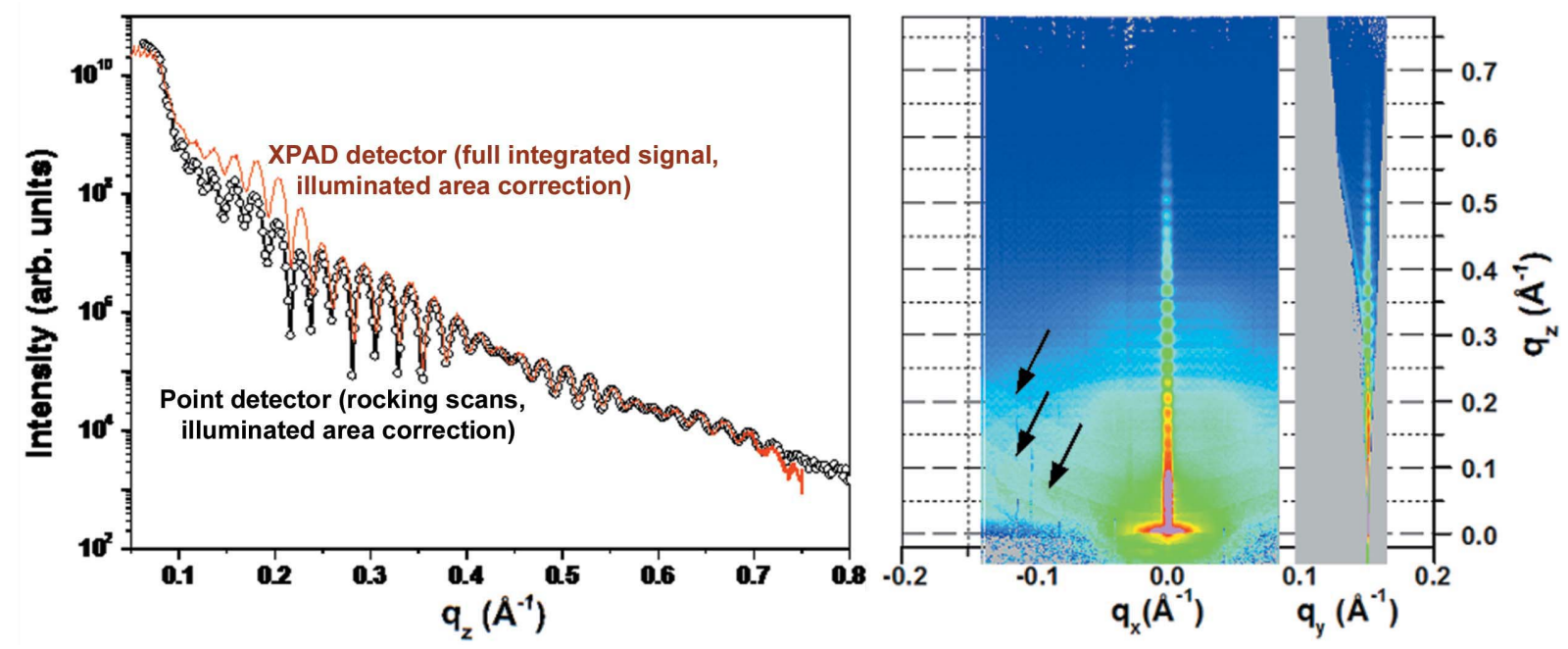

Figure 2

Soliton sample described in $\$ 2.1$. (Left) Comparison of the reflectivity curves acquired with a point detector (rocking scan) and fully integrated $q$-space converted XPAD images. Both data sets were corrected for the active (illuminated) area of the sample, and normalized for the same intensity at the total external reflection plateau. (Right) Two-dimensional cuts through the three-dimensional data set (after $q$-space reconstruction). Note that, in the figure, the horizontal $q_{y}$ axis length is expanded by a factor of 2 with respect to the vertical one (see the length of $0.1 \AA^{-1}$ scale in the horizontal and vertical directions). Also the $q_{x}$ coordinate was laterally shifted for display purposes. Note the presence of attenuators in the beam when measuring data close to the total external reflection of the beam (arrows). The intensity colour scale is logarithmic and spans over five orders of magnitude, from blue to red. 
is too large to intercept properly the high-symmetry directions in the reciprocal space (GISAXS signal) in a single area detector image. Alternative approaches were thus proposed (Lu et al., 2013; Yan \& Gibaud, 2007). Three-dimensional mapping of the reciprocal space allows one here to overcome in an elegant way this geometrical limitation; as a matter of fact, accurate cuts along high-symmetry planes can be easily obtained from the three-dimensional data set. The GISAXS data can be modelled (Lazzari, 2002; Stepanov, 2017) in order to extract meaningful information about the sample morphology. In the case of very low scattering in this region needing large exposure times, a motorized beamstop can be placed in front of the area detector to block the intense XRR signal and allow removal of beam attenuators and/or larger counting times to record with better statistics the low diffuse scattering signal.

An example of a GISAXS measurement performed as detailed above is shown in Fig. 2. The information contained in the GISAXS image (cut at $q_{x}=0$, containing as well the XRR curve) is richer than just the XRR curve. We can note the presence of structured diffuse scattered intensity for values of $q_{x} \sim \pm 0.02 \AA^{-1}$ (Fig. 2, right panel) corresponding to a realspace distance of about 25 to $30 \mathrm{~nm}$. Cross sections of the sample were also examined in high-resolution transmission electron microscopy (HR-TEM) (Fig. 3). The thickness of the different layers is confirmed, as well as a columnar growth of the film. It remains difficult to estimate the lateral size of these columns from the TEM images, but it would remain compatible with the lateral size of $30 \mathrm{~nm}$ deduced from the GISAXS data.
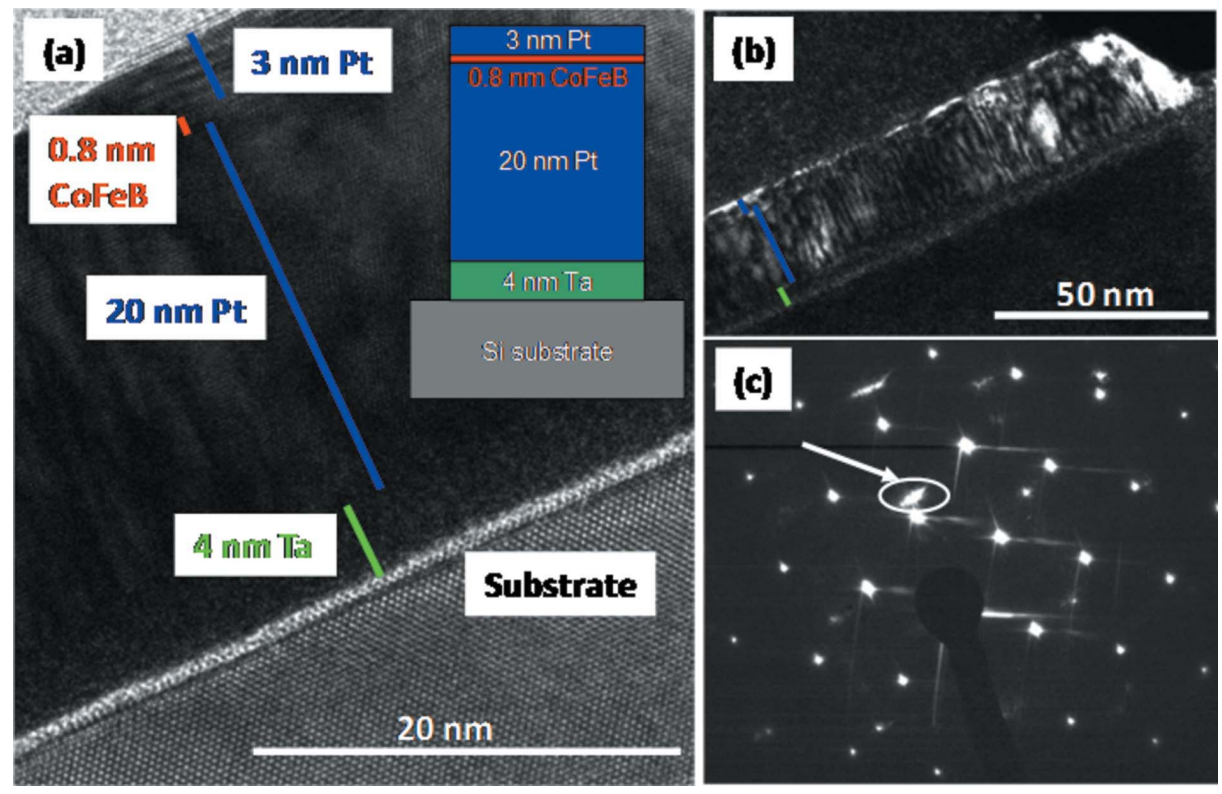

Figure 3

Soliton sample described in §2.1. (a) HR-TEM image; the inset details the sample structure as designed for manufacturing; $(b)$ dark-field (DF) image obtained micrograph realized using the diffraction spot marked in panel $(c)$ by the arrow - the spot is elongated in a direction parallel to the interface. The white zones in the image thus correspond to crystalline grains having the growth axis perpendicular to this interface (i.e. columnar growth). In both cases above, the coloured scale bars correspond to the expected thickness of the various layers present in the sample. The same coloured code as in the inset of panel $(a)$ is used. $(c)$ Electron diffraction pattern originating from the sample.
The XPAD was used to acquire data in continuous acquisition mode with different exposure times per image. Reliable data up to values of $q \simeq 0.6-0.7 \AA^{-1}$ can be obtained for exposure times as short as $10 \mathrm{~ms}$ per image: the total acquisition time of the full XRR data (in three dimensions, and including motor movements and beam attenuator insertion and extraction) is about $15 \mathrm{~s}$. The reciprocal-space volume can be reconstructed in all these cases - an example of the result obtained for the fastest acquisition is shown in the supporting information (Fig. S4). A measurement of the soliton sample was also performed using the XPAD detector and an X-ray beam of $19 \mathrm{keV}$ energy. The advantages of a proper setting of the detector discrimination threshold (Mocuta et al., 2013; Medjoubi et al., 2010, 2012) are counter-balanced by a poorer $q$-space resolution: as the detector is placed at the same distance with respect to the sample, the $q$-space resolution is reduced by a factor of about 2 by the X-ray energy increase. Despite this, the data obtained have a quality comparable with that of the data already shown in this paper.

A visual comparison of the data obtained on this soliton sample using either the point or area detector shows rather similar scattered intensity variation versus $q$ coordinate, but with some differences for $q \simeq 0.1$ to $0.25 \AA^{-1}$ (Fig. 2, right panel). A clear explanation of the origin of this difference between the two data sets cannot be given. We can speculate that the presence of the diffuse scattered intensity [appearing as satellites at $\left.\left(q_{x}, q_{z}\right) \sim(0.03,0.15) \AA^{-1}\right]$ in the GISAXS map contributed to an overestimation of the background level subtracted from the XRR data when a point detector and rocking scans are used - this would yield a lower-intensity level of the XRR point-detectorcorrected signal in Fig. 2 (right panel) for $q \simeq 0.15 \AA^{-1}$. Attempts to model the XRR data (using the package SimulRefl developed at CEA Saclay, IRAMIS) yield, as expected, differences in the extracted quantities, be it thickness of the layers or the respective interfacial roughness - these differences remain small enough to be attributed to the inherent errors of the measurement (e.g. Poisson distribution on the absolute value of the measured intensity in each point). A full propagation of the measurement errors has not yet been done, although is possible (the geometry of the measurement is well known and can be modelled in both above-mentioned cases). Moreover, the complexity of the soliton sample, the quality of the layers and their rather small thickness (see e.g. the presence of the very thin $0.8 \mathrm{~nm}$ CoFeB layer) do not allow for a clear choice of the better data set after comparing for example with TEM results. 
In an attempt to quantitatively determine the quality of the data using these two approaches, a rapid evaluation was made by performing a re-binning of the data on a regular $q$ step, followed by a fast Fourier transform (FFT) on each of the XRR data sets. The results show that the data obtained using the area detector have, even for the very low exposure times, better defined peaks attributed to thicknesses corresponding to the constituent layers (or summation of them) in the soliton sample (Fig. S5). A second example is the peculiar behaviour of an oxide thin film sample in the X-ray beam (of $8.0 \mathrm{keV}$ energy): the XRR interference pattern evolves while the sample is exposed to photons. Before detailing the results, we will point out that the effect seems to be triggered only at very high photon flux densities, such as those available at thirdgeneration synchrotron sources with well collimated and focused X-ray beams. Consequently, particular care is taken to align the sample in the highly attenuated X-ray beam, before acquiring reference XRR data (low photon flux); this reveals very broad and low-amplitude oscillations, which are manifested as destructive interferences (nodes) in the XRR curve. They are attributed to a very thin layer having a different density compared with the rest of the sample, which ensures the contrast needed to create this interference in the measured XRR signal. By removing all the beam attenuators and immediately acquiring (continuously and rapidly) XRR data, i.e. with increasing the $\mathrm{X}$-ray exposure of the sample, this broad oscillation becomes narrower: the node position moves towards lower $|\mathbf{q}|$ values and supplementary nodes (second and third order) appear subsequently at higher $|\mathbf{q}|$ (or $2 \theta$ ) values. This is illustrated in Fig. 4 (left panel, arrows). The period of this broad oscillation is more than ten times that originating from the thin film layer, about $10 \mathrm{~nm}$, and therefore associated with a thin layer (less than $1 \mathrm{~nm}$ thickness). The amplitude and the contrast of this broad oscillation in the XRR curve are very low, and thus associated with a slightly
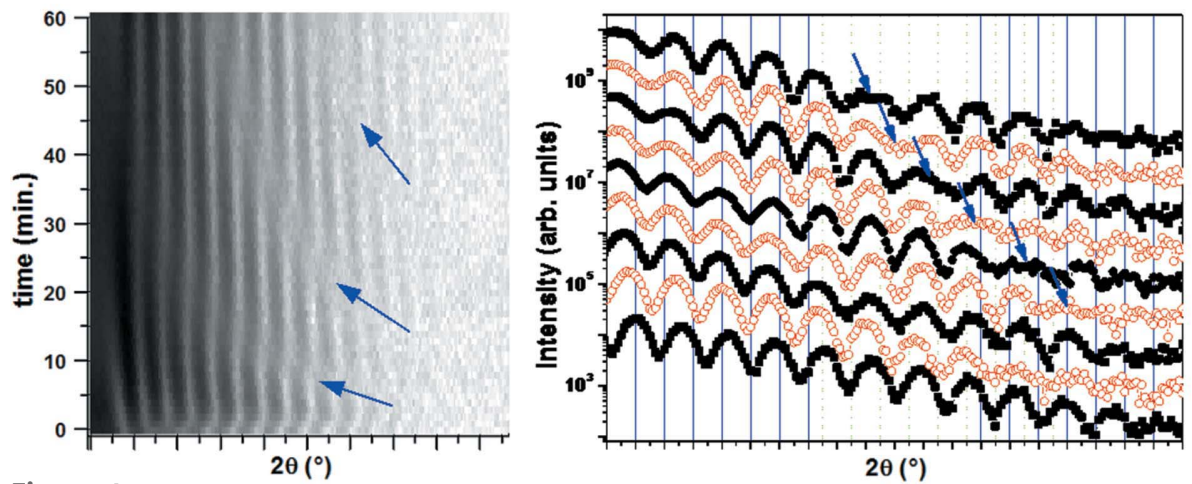

Figure 4 $2 \theta\left(^{\circ}\right)$

$\mathrm{BaTiO}_{3}$ thin layer sample described in $\$ 2.2$. (Left) Fast XRR curves (partial data, the total externa reflection plateau is not shown) measured every minute for the sample exposed to the X-ray beam. One measurement took about $30 \mathrm{~s}$. An evolution of the sample in the X-ray beam can be noticed: the arrows point out changes in the position of a broad supplementary oscillation appearing in the data, and moving through them during the X-ray exposure. The intensity colour scale is logarithmic and spans over three orders of magnitude, from white to black. (Right) Comparison of several XRR data recorded at different moments in time, extracted from the previous data set: the curves correspond to data recorded every $\sim 5 \mathrm{~min}$. Besides the displacements of the intensity minima, the regions highlighted by arrows show the presence and displacement of a negative interference effect moving towards lower $2 \theta$ values. This effect and its origin will be discussed elsewhere. different electron density. It is only by using the extremely high photon flux available at a synchrotron source (and access to large momentum transfer values) that one is able to show these low-amplitude and broad oscillations. Moreover, measuring three-dimensional data sets and integrating the scattered signal, including background correction, allow this very low amplitude signal to be highlighted, which might be overlooked in a measurement with a point detector - most of the time this oscillation translates into a very faded signal.

We should point out here that the photon flux density varies slightly on the sample during the XRR measurement, due to the X-ray beam footprint changing on the sample. Also, the probed area of the sample surface changes accordingly. We should also mention that in a classical XRR measurement with onochromatic X-rays it is expected that a possible sample olution in the beam (i.e. XRR signal changing in time) will 'smoothed out' by the measurement approach itself. The oint detector approach, either a linear $q_{z}$ scan or inte(rocking) approach] is generally long enough to bring 列 changes in the X-ray beam. Even if it the 'initial' state of the sample for the low 'final' state (modified by the X-ray beam exposure) for large $q$ values. The transition will not be obvious in the XRR curve. From the qualitative measurements, we observe evolutions but we cannot make quantitative measurement using the classical XRR data acquisition scheme. We ruled out any artifact related to the experimental approach by several tests and measurement campaigns, using various setups and samples. The details will be reported elsewhere.

The third example shows the formation of a diffuse nickel silicide layer as a result of a solid-state reaction during the thermal annealing of a thin film of $\mathrm{Ni}$ deposited on a $\mathrm{Si}(001)$ substrate, similarly to what was reported by Putero et al. (2010, 2013), Ehouarne et al. (2006). The sample was mounted on an Anton Paar heater (https:// www.anton-paar.com/corp-en/) (model DHS 900), under a poly-ether-etherketone (PEEK) dome; the annealing is performed under a $\mathrm{He}$ atmosphere. Once the sample was aligned in the X-ray beam, fast XRR measurements (X-ray beam energy of $8.0 \mathrm{keV}$ ) are launched in an endless loop while heating the sample to $290^{\circ} \mathrm{C}$ with a $6^{\circ} \mathrm{C} \mathrm{m^{-1 }}$ ramp in temperature. The resulting data are reported in Fig. 5: at each point, the absolute time and the heater temperature are recorded. Measuring a single reflectivity curve is achieved in less than $20 \mathrm{~s}$. The measurements were also completed by wide-angle diffraction ones in a $\theta-2 \theta$ geometry (Fig. 5, right panel), which 
were performed on the sample during the thermal annealing, under the very same experimental conditions. This approach could be easily extended to measure/characterize the system via other fast acquisition methods (Medjoubi, Bonissent et al., 2013; Medjoubi, Leclercq et al., 2013; Leclercq et al., 2015; Chahine et al., 2014), including strain and texture measurements (Fouet et al., 2012; Gaudet et al., 2013; Mocuta et al., 2013; Richard et al., 2013, 2015).

Several temperature regions can be distinguished from these measurements (Fig. 5). We will try to point out the main features:

(i) Temperatures up to $\sim 100^{\circ} \mathrm{C}$ : no significant changes can be distinguished, either in XRR or in the X-ray diffraction (XRD) data. The slight shift of the Ni(111) Bragg peak position towards lower $2 \theta$ values is characteristic of the thermal expansion of the lattice.

(ii) $100-180^{\circ} \mathrm{C}$ : the Ni layer is slowly consumed (intensity of the corresponding Bragg peak is slowly diminishing) while the creation of a new layer is seen in the XRR data (appearance of interference). The shift of the $\mathrm{Ni}(111)$ Bragg peak position in XRD towards higher $2 \theta$ values could be attributed to a relaxation of the lattice parameter in the thinner film.

(iii) $180-210^{\circ} \mathrm{C}$ : rapid changes are detected in the XRR signal. The faster decay of the XRR signal at large scattering angles could point towards a roughening of the layer. The value of the critical angle for total external reflection slightly decreases, which is in agreement with a significant inclusion of $\mathrm{Si}$ into the Ni layer. Thickness oscillations are fading and becoming broader in XRD data.

(iv) $210-290^{\circ} \mathrm{C}$ : very rapid consumption of the $\mathrm{Ni}$ layer (cf. XRD), as well as a rapid appearance of nickel silicide peaks - their intensity seems to stabilize rapidly. XRR signals seem to extend further away in reciprocal space than in (iii), with rapid changes of the interference pattern versus the temperature.

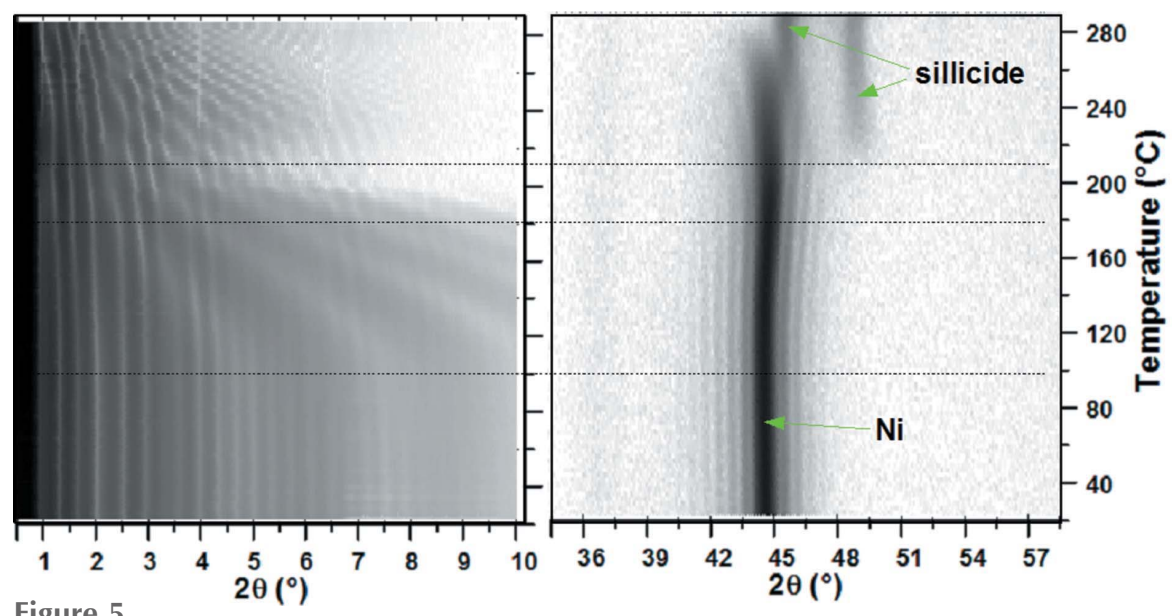

Figure 5 $2 \theta\left({ }^{\circ}\right)$

$\mathrm{Ni}$ thin layer sample, detailed in §2.3. (Left) Temperature evolution of the XRR signal recorded (every $\sim 30 \mathrm{~s}$ ) during the in situ thermal annealing of the sample. The broad oscillations giving extra contrast (period of $\sim 2^{\circ}$ in $2 \theta$ for low temperatures) correspond to the topmost capping layer. (Right) Temperature evolution of the XRD signal recorded under the same conditions, during the in situ thermal annealing of the sample. The colour scale is logarithmic in both cases and spans over nine (left panel) and four (right panel) orders of magnitude, from white to black. Several temperature regimes can be distinguished, which are discussed in more detail in the text.
Higher annealing temperatures could have been used; the nickel silicidation and creation of interesting phases are expected for temperatures above $300^{\circ} \mathrm{C}$. We point out here that, even if a wealth of information can be extracted from these data [approaches to extract information from the large number of XRR curves have already been proposed (Putero et al., 2010, 2013; Ehouarne et al., 2006)], it was not the aim of this paper to detail and understand the creation of this diffuse interface - the corresponding results will be shown elsewhere.

\section{Conclusion}

We have shown here a genuine experimental approach possible at modern synchrotron sources, to perform fast threedimensional reciprocal-space mapping (several to $10 \mathrm{~s}$ ) in the vicinity of the origin of the reciprocal space, and extract XRR and GISAXS information. The approach uses fast-reading and low-noise area detectors and synchronous motor movements. At high-intensity X-ray sources, the total acquisition time limits are fixed by the combination of the maximal motor speeds and the desired area detector acquisition speed (limiting exposure time per frame). After proving the concept on a model sample, we illustrated our method using a sample exhibiting an evolution in the X-ray beam with dynamics over minutes, and thus compatible with the characteristic acquisition times of the fast XRR approach detailed here. In the last example, we investigated the thermal kinetics (formation of a diffuse interface) during an annealing process. This approach reveals its usefulness for studying processes like rapid thermal annealing or quenching.

Compared with the fast XRR approaches developed before by other groups (and mentioned at the beginning of this paper), we can point out both advantages and drawbacks. Other approaches ensure better time resolutions, with values down to as low as $100 \mathrm{ps}$ being reported in a pump-probe experiment (Nüske et al., 2011); in this case, the structural changes need to be reversible (with fast relaxation times). But in most of the cases line-like XRR data (possibly with an offset background line for correction) can be extracted. Our approach ensures a three-dimensional mapping of the reciprocal space; later on, different data sets can be extracted (for example, by choosing particular ROIs in the full data set), which is impossible on integrated data acquired using standard XRR acquisition schemes with a point detector. There is also the possibility to easily tune the $q$-space resolution element or the measured $q$ range. The energy of the X-rays during the measurement can also be chosen to avoid getting closer to absorption edges and deal with fluorescence parasitic background. The recorded data set contains, without the need for any extra acquisi- 
tion, the GISAXS signal, which can be important in understanding the sample structure and its morphology.

Compared with the approach of the fast rotating sample mounted on a wedge (Buffet et al., 2013), our approach has the advantage of always illuminating the same sample region (i.e. same strip along the X-ray beam direction), which would not be the case for a rotating sample. In the case of the wedge sample, the resulting $q$-space voxels yield a non-uniform $q$-space grid, so a proper integration of the data set might become more complicated. The $\theta-2 \theta$ continuous scanning approach results in data sets with a more regular $q$-space gridding, which are easier to regroup in order to compare with point detector acquisitions. It also offers the flexibility of cutting the XRR curve into several ranges, each of them measured with the proper X-ray beam attenuator to ensure a linear response of the detector. Sample environments could also be less compatible with a fast and endless rotating sample setup. Depending on the phenomena to be studied and the characteristic time scales, an approach that is appropriate and compatible (with the setup and the sample environment) will have to be considered from those available.

Characterizing the morphology of a thin film sample and its time evolution with a resolution of less than $10 \mathrm{~s}$ might reveal its importance in studying phenomena like the absorption of proteins at solid-liquid interfaces (time scales between seconds and several minutes), thermal annealing and phase transformation or sample changes in the X-ray beam - the last two as shown in this paper. The approach depicted here also paves the way to accessing dynamics in the crystalline structure of samples, a topic which will definitely gain interest in the next few years.

\section{Related literature}

The following references, not cited in the main body of the paper, have been cited in the supporting information: Abboud et al. (2011); Albouy \& Valerio (1997); Als Nielsen \& McMorrow (2010); Bhattacharya et al. (2003); Brower et al. (1996); Chihab \& Naudon (1992); Daillant \& Alba (2000); Daillant \& Gibaud (2009); Fenter, Catalano et al. (2006); Fenter, Park et al. (2006); Holy et al. (1999); Jibaoui \& Erre (2001); Kozhevnikov et al. (2008); Laanait et al. (2014); Lueken et al. (1994); Matsushita et al. (2008, 2013); Metzger et al. (1994); Mizusawa \& Sakurai (2011); Murphy et al. (2014); Nakano et al. (1978); Naudon et al. (1989); Niggemeier et al. (1997); Omote et al. (2000, 2001); Parratt (1954); Peverini et al. (2007); Sakurai (2004); Sakurai, Mizusawa \& Ishi (2007); Sakurai, Mizusawa, Ishi, Kobayashi et al. (2007); Sato et al. (2000); Seeck (2014); Stoev \& Sakurai (1999, 2013); Tolan (1999); Vlieg (1997); Voegeli et al. (2013); Wirkert et al. (2013); Yasaka (2010).

\section{Acknowledgements}

All the data reported in this paper were recorded at Synchrotron SOLEIL (France) on the DiffAbs beamline. F. Alves, P. Joly and P. Monteiro are acknowledged for their help with the experimental setup. A. Fernandez-Pacheco from the Cavendish Laboratory, University of Cambridge, is acknowledged for providing the soliton sample. The oxide team from CEA Saclay is acknowledged for excellent working conditions on the Oxygen Assisted Molecular Beam Epitaxy and for supplying the oxide samples. C. Lavoie from IBM T. J. Watson Research Center and A. S. Özcan from ST Microelectronics (IBM team) are acknowledged for supplying the $\mathrm{Ni} / \mathrm{Si}$ sample.

\section{References}

Abboud, A., Send, S., Hartmann, R., Strüder, L., Savan, A., Ludwig, A., Zotov, N. \& Pietsch, U. (2011). Phys. Status Solidi A, 208, 26012607.

Albouy, P.-A. \& Valerio, P. (1997). Supramol. Sci. 4, 191-194.

Als Nielsen, J. \& McMorrow, D. (2010). Elements of Modern X-ray Physics, 2nd ed. New York: John Wiley and Sons.

Barbier, A., Aghavnian, T., Badjeck, V., Mocuta, C., Stanescu, D., Magnan, H., Rountree, C. L., Belkhou, R., Ohresser, P. \& Jedrecy, N. (2015). Phys. Rev. B, 91, 035417.

Barbier, A., Mocuta, C. \& Belkhou, R. (2012). Selected Synchrotron Radiation Techniques, Encyclopedia of Nanotechnology, Vol. 19, edited by B. Bhushan, pp. 2322-2344. The Netherlands: Springer.

Barbier, A., Mocuta, C., Stanescu, D., Jegou, P., Jedrecy, N. \& Magnan, H. (2012). J. Appl. Phys. 112, 114116.

Basolo, S. et al. (2007). J. Synchrotron Rad. 14, 151-157.

Basolo, S. et al. (2008). Nucl. Instrum. Methods Phys. Res. A, 589, 268-274.

Bhattacharya, M., Mukherjee, M., Sanyal, M. K., Geue, Th., Grenzer, J. \& Pietsch, U. (2003). J. Appl. Phys. 94, 2882-2887.

Breil, N., Lavoie, C., Ozcan, A., Baumann, F., Klymko, N., Nummy, K., Sun, B., Jordan-Sweet, J., Yu, J., Zhu, F., Narasimha, S. \& Chudzik, M. (2015). Microelectron. Eng. 137, 79-87.

Brower, D. T., Revay, R. E. \& Huang, T. C. (1996). Powder Diffr. 11, 114-116.

Buffet, A., Lippmann, M., Pflaum, K. \& Seeck, O. H. (2013). Photon Science 2013 - Highlights and Annual Report, pp. 102-103. DESY, Hamburg, Germany.

Chahine, G. A., Richard, M.-I., Homs-Regojo, R. A., Tran-Caliste, T. N., Carbone, D., Jacques, V. L. R., Grifone, R., Boesecke, P., Katzer, J., Costina, I., Djazouli, H., Schroeder, T. \& Schülli, T. U. (2014). J. Appl. Cryst. 47, 762-769.

Chihab, J. \& Naudon, A. (1992). J. Phys III (Fr.), 2, 2291-2300.

Daillant, J. \& Alba, M. (2000). Rep. Prog. Phys. 63, 1725-1777.

Daillant, J. \& Gibaud, A. (2009). X-ray and Neutron Reflectivity. Berlin: Springer.

Delpierre, P. et al. (2007). Nucl. Instrum. Methods Phys. Res. A, 572, 250-253.

Ehouarne, L., Putero, M., Mangelinck, D., Nemouchi, F., Bigault, T., Ziegler, E. \& Coppard, R. (2006). Microelectron. Eng. 83, 22532257.

Fenter, P., Catalano, J. G., Park, C. \& Zhang, Z. (2006). J. Synchrotron Rad. 13, 293-303.

Fenter, P., Park, C., Zhang, Z. \& Wang, S. (2006). Nat. Phys. 2, 700 704.

Fertey, P., Alle, P., Wenger, E., Dinkespiler, B., Cambon, O., Haines, J., Hustache, S., Medjoubi, K., Picca, F., Dawiec, A., Breugnon, P., Delpierre, P., Mazzoli, C. \& Lecomte, C. (2013). J. Appl. Cryst. 46, 1151-1161.

Fouet, J., Richard, M.-I., Mocuta, C., Guichet, C. \& Thomas, O. (2012). Nucl. Instrum. Methods Phys. Res. B, 284, 74-77.

Fujii, Y. (2010). Surf. Interface Anal. 42, 1642-1645.

Fujii, Y. (2013). Powder Diffr. 28, 100-104.

Gaudet, S., De Keyser, K., Lambert-Milot, S., Jordan-Sweet, J., Detavernier, C., Lavoie, C. \& Desjardins, P. (2013). J. Vac. Sci. Technol. A, 31, 021505. 
Holy, V., Pietsch, U. \& Baumbach, T. (1999). High Resolution X-ray Scattering from Thin Films and Multilayers. Berlin: Springer.

Imbert, B., Pantel, R., Zoll, S., Gregoire, M., Beneyton, R., del Medico, S. \& Thomas, O. (2010). Microelectron. Eng. 87, 245-248. Jibaoui, H. \& Erre, D. (2001). Surf. Rev. Lett. 8, 11-17.

Kittl, J. A., Lauwers, A., Chamirian, O., Van Dal, M., Akheyar, A., De Potter, M., Lindsay, R. \& Maex, K. (2003). Microelectron. Eng. 70, 158-165.

Kobayashi, S. \& Inaba, K. (2012). Rigaku J. 28, 8-13.

Kozhevnikov, I., Peverini, L. \& Ziegler, E. (2008). Opt. Express, 16, 144-149.

Laanait, N., Zhang, Z., Schlepütz, C. M., Vila-Comamala, J., Highland, M. J. \& Fenter, P. (2014). J. Synchrotron Rad. 21, 1252-1261.

Lauwers, A., Kittl, J. A., Van Dal, M. J. H., Chamirian, O., Pawlak, M. A., de Potter, M., Lindsay, R., Raymakers, T., Pages, X., Mebarki, B., Mandrekar, T. \& Maex, K. (2004). Mater. Sci. Eng. B, 114-115, 29-41.

Lavoie, C., d'Heurle, F. M., Detavernier, C. \& Cabral Jr, C. (2003). Microelectron. Eng. 70, 144-157.

Lavrijsen, R., Lee, J., Fernández-Pacheco, A., Petit, D. C. M. C., Mansell, R. \& Cowburn, R. P. (2013). Nature, 493, 647-650.

Lazzari, R. (2002). J. Appl. Cryst. 35, 406-421.

Le Bourlot, C., Landois, P., Djaziri, S., Renault, P.-O., Le Bourhis, E., Goudeau, P., Pinault, M., Mayne-L'Hermite, M., Bacroix, B., Faurie, D., Castelnau, O., Launois, P. \& Rouzière, S. (2012). J. Appl. Cryst. 45, 38-47.

Leclercq, N., Berthault, J., Langlois, F., Le, S., Poirier, S., Bisou, J., Blache, F., Medjoubi, K. \& Mocuta, C. (2015). 15th International Conference on Accelerator and Large Experimental Physics Control Systems (ICALEPCS), Melbourne, Australia.

Lu, X., Yager, K. G., Johnston, D., Black, C. T. \& Ocko, B. M. (2013). J. Appl. Cryst. 46, 165-172.

Lueken, E., Ziegler, E., Hoeghoej, P., Freund, A. K., Gerdan, E. \& Fontaine, A. (1994). International Symposium on Optical Interference Coatings, doi: 10.1117/12.192085.

Matsushita, T., Arakawa, E., Voegeli, W. \& Yano, Y. F. (2013). J. Synchrotron Rad. 20, 80-88.

Matsushita, T., Niwa, Y., Inada, Y., Nomura, M., Ishii, M., Sakurai, K. \& Arakawa, E. (2008). Appl. Phys. Lett. 92, 024103.

Medjoubi, K., Bonissent, A., Leclercq, N., Langlois, F., Mercère, P. \& Somogyi, A. (2013). Proc. SPIE, 8851, 8851OP.

Medjoubi, K., Bucaille, T., Hustache, S., Bérar, J.-F., Boudet, N., Clemens, J.-C., Delpierre, P. \& Dinkespiler, B. (2010). J. Synchrotron Rad. 17, 486-495.

Medjoubi, K., Leclercq, N., Langlois, F., Buteau, A., Lé, S., Poirier, S., Mercère, P., Sforna, M. C., Kewish, C. M. \& Somogyi, A. (2013). J. Synchrotron Rad. 20, 293-299.

Medjoubi, K. et al. (2012). J. Synchrotron Rad. 19, 323-331.

Metzger, T. H., Luidl, C., Pietsch, U. \& Vierl, U. (1994). Nucl. Instrum. Methods Phys. Res. A, 350, 398-405.

Mizusawa, M. \& Sakurai, K. (2011). Mater. Sci. Eng. 24, 012013.

Mocuta, C., Richard, M.-I., Fouet, J., Stanescu, S., Barbier, A., Guichet, C., Thomas, O., Hustache, S., Zozulya, A. V. \& Thiaudière, D. (2013). J. Appl. Cryst. 46, 1842-1853.

Mocuta, C., Stangl, J., Mundboth, K., Metzger, T. H., Bauer, G., Vartanyants, I. A., Schmidbauer, M. \& Boeck, T. (2008). Phys. Rev. $B, \mathbf{7 7}, 245425$.

Murphy, B. M., Greve, M., Runge, B., Koops, C. T., Elsen, A., Stettner, J., Seeck, O. H. \& Magnussen, O. M. (2014). J. Synchrotron Rad. 21, 45-56.

Nakano, Y., Fukamachi, T. \& Hayakawa, K. (1978). Jpn. J. Appl. Phys. 17, 329-331.

Naudon, A., Chihab, J., Goudeau, P. \& Mimault, J. (1989). J. Appl. Cryst. 22, 460-464.

Niggemeier, U., Lischka, K., Plotz, W. M. \& Holy, V. (1997). J. Appl. Cryst. 30, 905-908.
Nüske, R., Jurgilaitis, A., Enquist, H., Farahani, S. D., Gaudin, J., Guerin, L., Harb, M., Korff Schmising, C. v., Störmer, M., Wulff, M. \& Larsson, J. (2011). Appl. Phys. Lett. 98, 101909.

Omote, K., Kikuchi, T., Harada, J., Kawasaki, M., Ohtomo, A., Ohtani, M., Ohnishi, T., Komiyama, D. \& Koinuma, H. (2000). Proc. SPIE, 3941, 84-91.

Omote, K., Kikuchi, T., Harada, J., Kawasaki, M., Ohtomo, A., Ohtani, M., Ohnishi, T., Komiyama, D. \& Koinuma, H. (2001). Rigaku J. 18, 38-45.

Paciorek, W. A., Meyer, M. \& Chapuis, G. (1999a). Acta Cryst. A55, 543-557.

Paciorek, W. A., Meyer, M. \& Chapuis, G. (1999b). J. Appl. Cryst. 32, 11-14.

Pangaud, P., Basolo, S., Boudet, N., Berar, J., Chantepie, B., Clemens, J., Delpierre, P., Dinkespiler, B., Medjoubi, K., Hustache, S., Menouni, M. \& Morel, C. (2008). Nucl. Instrum. Methods Phys. Res. A, 591, 159-162.

Pangaud, P., Basolo, S., Boudet, N., Berar, J.-F., Chantepie, B., Delpierre, P., Dinkespiler, B., Hustache, S., Menouni, M. \& Morel, C. (2007). Nucl. Instrum. Methods. A, 571, 321-324.

Parratt, L. G. (1954). Phys. Rev. 95, 359-369.

Peverini, L., Kozhevnikov, I. \& Ziegler, E. (2007). Phys. Status Solidi A, 204, 2785-2791.

Putero, M., Coulet, M.-V., Ouled-Khachroum, T., Muller, C., Baehtz, C. \& Raoux, S. (2013). APL Mater. 1, 062101.

Putero, M., Ehouarne, L., Ziegler, E. \& Mangelinck, D. (2010). Scr. Mater. 63, 24-27.

Renaud, G., Lazzari, R. \& Leroy, F. (2009). Surf. Sci. Rep. 64, 255380.

Richard, M.-I., Fouet, J., Guichet, C., Mocuta, C. \& Thomas, O. (2013). Thin Solid Films, 530, 100-104.

Richard, M.-I., Fouet, J., Texier, M., Mocuta, C., Guichet, C. \& Thomas, O. (2015). Phys. Rev. Lett. 115, 266101.

Sakurai, K. (2004). X-ray Spectrometry: Recent Technological Advances. New York: J. Wiley.

Sakurai, K., Mizusawa, M. \& Ishii, M. (2007). Trans. MRS Jpn, 32, 181-186.

Sakurai, K., Mizusawa, M., Ishii, M., Kobayashi, S. \& Imai, Y. (2007). J. Phys. Conf. Ser. 83, 012001.

Sato, S., Imanaga, T., Matsubara, E., Saito, M. \& Waseda, Y. (2000). Mater. Trans. JIM, 41, 1651-1656.

Schlepütz, C. M., Herger, R., Willmott, P. R., Patterson, B. D., Bunk, O., Brönnimann, Ch., Henrich, B., Hülsen, G. \& Eikenberry, E. F. (2005). Acta Cryst. A61, 418-425.

Seeck, O. (2014). Z. Phys. Chem. 228, 1135-1154.

Sinha, S. K., Sirota, E. B., Garoff, S. \& Stanley, H. B. (1988). Phys. Rev. B, 38, 2297-2311.

Stanescu, S., Mocuta, C., Merlet, F. \& Barbier, A. (2013). J. Synchrotron Rad. 20, 181-189.

Stepanov, S. (2017). X-ray server (TER-sl, TRDS_sl), http:// sergey.gmca.aps.anl.gov/ [last accessed: 09/10/2017].

Stoev, K. N. \& Sakurai, K. (1999). At. Spectrosc. 54, 41-82.

Stoev, K. \& Sakurai, K. (2013). Powder Diffr. 28, 105-111.

Tolan, M. (1999). X-ray Scattering from Soft Matter Thin Films, Materials Science and Basic Research, Vol. 148 of Springer Tracts in Modern Physics. Berlin: Springer.

Vlieg, E. (1997). J. Appl. Cryst. 30, 532-543.

Voegeli, W., Matsushita, T., Arakawa, E., Shirasawa, T., Takahashi, T. \& Yano, Y. F. (2013). J. Phys. Conf. Ser. 425, 092003.

Weber, W. \& Lengeler, B. (1992). Phys. Rev. B, 46, 7953-7956.

Wirkert, F. J., Paulus, M., Sternemann, C., Nase, J., Schroer, M. A., Wieland, D. C. F., Bieder, S., Degen, P., Rehage, H. \& Tolan, M. (2013). J. Phys. Conf. Ser. 425, 202006.

Xu, B., Yin, K. B., Lin, J., Xia, Y. D., Wan, X. G., Yin, J., Bai, X. J., Du, J. \& Liu, Z. G. (2009). Phys. Rev. B, 79, 134109.

Yan, M. \& Gibaud, A. (2007). J. Appl. Cryst. 40, 1050-1055.

Yasaka, M. (2010). Rigaku J. 26, 1-9. 